\title{
Duration reproduction in regular and irregular contexts after unilateral brain damage: Evidence from voxel-based lesion-symptom mapping and atlas-based hodological analysis
}

\author{
Alice Teghil $^{\text {a, }}$, Antonella Di Vita ${ }^{\mathrm{a}, \mathrm{b}}$, Veronica Pietranelli $^{\mathrm{b}}$, Alessandro Matano ${ }^{\mathrm{c}}$, \\ Maddalena Boccia ${ }^{\mathrm{b}, \mathrm{a}}$ \\ ${ }^{a}$ Cognitive and Motor Rehabilitation and Neuroimaging Unit, IRCCS Fondazione Santa Lucia, Rome, Italy \\ b Department of Psychology, "Sapienza" University of Rome, Rome, Italy \\ ${ }^{\mathrm{c}}$ Neuropsychology Unit, IRCCS Fondazione Santa Lucia, Rome, Italy
}

\section{A R T I C L E I N F O}

\section{Keywords:}

Time perception

Brain damage

Voxel lesion symptom mapping

Context

\begin{abstract}
A B S T R A C T
It has been proposed that not completely overlapping brain networks support interval timing depending on whether or not an external, predictable temporal cue is provided during the task, aiding time estimation. Here we tested this hypothesis in a neuropsychological study, using both a topological approach - through voxel-based lesion-symptom mapping (VLSM), that assesses the relation between continuous behavioral scores and lesion information on a voxel-by-voxel basis - and a hodological approach, using an atlas-based tractography. A group of patients with unilateral focal brain lesions and their matched controls performed a duration reproduction task assessing time processing in two conditions, namely with regularly spaced stimuli during encoding and reproduction (Regular condition), and with irregularly spaced stimuli during the same task (Irregular condition). VLSM analyses showed that scores in the two conditions were associated with lesions involving partly separable clusters of voxels, with lower performance only in the Irregular condition being related to lesions involving the right insular cortex. Performance in both conditions correlated with the probability of disconnection of the right frontal superior longitudinal tract, and of the superior and middle branches of the right superior longitudinal fasciculus. These findings suggest that the dissociation between timing in regular and irregular contexts is not complete, since performance in both conditions relies on the integrity of a common suprasecond timing network. Furthermore, they are consistent with the hypothesis that tracking time without the aid of external cues selectively relies on the integration of psychophysiological changes in the right insula.
\end{abstract}

\section{Introduction}

A temporal dimension is intrinsic to almost every type of cognitive operation and process. The ability to represent the duration of events in the seconds-to-minutes range (interval timing, Buhusi and Meck, 2005), specifically, is essential to most complex behaviors, and is a key prerequisite to high-level cognitive processes such as problem-solving, planning, and memory. However, despite neuroscientific research on time perception has grown considerably over the last twenty years, how the brain represents temporal features of experience is still not yet completely understood.

The study of alterations of interval timing following focal brain damage and neurodegenerative disorders has provided important insights into the neural substrates of the representation of both subsecond and suprasecond durations. Consistently with evidence from neuroimaging studies, pointing to the involvement of multiple brain regions in timing (for reviews, see Lewis and Miall, 2003a; Wiener et al., 2010a; Teghil et al., 2019), this body of literature has shown that duration processing deficits may arise from lesions to different subcortical and cortical structures.

Concerning the contribution of subcortical structures to duration processing, a role of the basal ganglia in timing is supported by studies on Parkinson's Disease patients. These patients show impaired sensorimotor synchronization, also independently from the motor component of performance (Harrington et al., 1998a; Freeman et al., 1993), as well as deficits in discriminating rhythmical tone sequences (Grahn and

\footnotetext{
* Corresponding author. Cognitive and Motor Rehabilitation and Neuroimaging Unit, IRCCS Fondazione Santa Lucia, via Ardeatina 306, 00179, Rome, Italy.

E-mail addresses: a.teghi@hsantalucia.it, alice.teghil@gmail.com (A. Teghil).
} 
Brett, 2009). Focal lesions of the basal ganglia also result in abnormal sensorimotor synchronization (Coslett et al., 2010) and reduced electrophysiological responses to regular compared to irregular tone sequences (Schwartze et al., 2015). Cerebellar lesions, as well, impair performance in motor timing tasks, such as sensorimotor synchronization (Schlerf et al., 2007; Schwartze et al., 2016), but also in perceptual ones, such as the discrimination of the duration of subsecond (Mangels et al., 1998; Grube et al., 2010) and suprasecond stimuli (Mangels et al., 1998), the bisection of time intervals (Nichelli et al., 1996), and the detection of changes in the structure (Molinari et al., 2003) and speed (Schwartze et al., 2016) of rhythms.

Studies on brain damaged patients have also highlighted the contribution of different cortical regions to timing. Lesions to the left and right dorsolateral prefrontal cortex have been shown to alter respectively the production and estimation of long time intervals (60-90 seconds) (Binkofsi and Block, 1996; Koch et al., 2002). Lesions to the orbitofrontal cortex also result in deficits in the production and estimation of multi-seconds time intervals (Berlin et al., 2004). The discrimination of shorter intervals (4-second), however, has also been found impaired in patients with lesions mainly overlapping in the lateral prefrontal cortex (Mangels et al., 1998), and time bisection in the subsecond range is impaired as well in patients with frontal brain lesions (Nichelli et al., 1995). Patients with right-sided lesions of the pars triangularis of the inferior frontal gyrus also show abnormal performance in both the synchronization and continuation phase of sensorimotor synchronization, whereas patients with left-sided lesions of the superior medial prefrontal cortex have been reported to be only impaired in the continuation phase (Picton et al., 2006).

There is also neuropsychological evidence for the involvement of parietal regions in timing. Reproduction accuracy for 2-second durations, as well as reproduction variability for longer durations (4-12second), have been found to correlate significantly with percent damage in the superior and inferior parietal lobe (Coslett et al., 2009). A key role of right-sided prefrontal and inferior parietal areas was also highlighted by Harrington and colleagues(1998a, 1998b), who tested interval discrimination of patients with focal left or right hemispheric cortical lesions in the subsecond range (Harrington et al., 1998b).

Though this body of evidence is in line with neuroimaging literature, consistently implicating the basal ganglia, the cerebellum, prefrontal and parietal regions in interval timing (Merchant et al., 2013; Koch et al., 2009), patient studies suggest that other cortical regions may also have some functional significance in duration processing. Indeed, the only voxel-based lesion-symptom mapping (VLSM) study investigating interval timing to date (Gooch et al., 2011) has shown an association between duration discrimination variability for visual stimuli, and lesions to the right inferior frontal gyrus, middle frontal gyrus, and precentral gyrus. Also, lesions to the left-sided basal ganglia, superior and middle temporal lobe, and hippocampus, were associated with performance variability specifically in the subsecond range (Gooch et al., 2011).

An altered perception of suprasecond durations has also been reported in neglect patients with lesions mainly overlapping in the right supramarginal gyrus, superior temporal gyrus, insula and basal ganglia (Danckert et al., 2007). Supporting a possible role of the insular cortex in timing, at least in specific conditions, interval reproduction accuracy in the suprasecond range has been found to be impaired in an epileptic patient with a focal lesion of the right anterior insular/inferior frontal cortex (Monfort et al., 2014).

Finally, there is also neuropsychological evidence of hippocampal involvement in timing. Patient H. M. showed impaired reproduction of durations ranging from 20 to 300 seconds, but normal performance for durations between 1 and 20 seconds (Richards, 1973). Patients with medial temporal lobe damage are also impaired in discriminating durations longer than 4 minutes (Palombo et al., 2016), and show reduced accuracy in interval production and estimation in the minute range (Noulhiane et al., 2007), suggesting that the hippocampus may be involved in interval timing in the minutes - rather than seconds - range (Palombo et al., 2016; Palombo and Verfaellie, 2017). In other studies, however, patients with medial temporal lobe lesions showed timing deficits also in the second (Melgire et al., 2005; Perbal et al., 2001) and subsecond range (Melgire et al., 2005), as well as in processing rhythmic stimuli (Ehrlé et al., 2001). Thus, it has been recently proposed that the hippocampus could be specifically involved in duration processing when the task entails sequential demands, independently from the duration range (Palombo et al., 2020; Lee et al., 2020).

Overall, the neuropsychological findings reviewed above suggest that duration processing relies on the integrity of multiple brain regions, consistently with accounts positing that different neural circuits play a differential role in timing depending on context and task features (Bueti et al., 2008a; Wiener et al., 2011a; Merchant et al., 2013; Wiener and Kanai, 2016). In this vein, previous literature has shown that different brain networks support timing depending on whether durations are perceived in absolute terms or relative to a regular beat (Teki et al., 2011), depending on the temporal range investigated (subsecond vs. suprasecond), on the degree of involvement of motor demands (Wiener et al., 2010a; Bueti et al., 2008b), and of attentional resources (Lewis and Miall, 2003a), and on the implicit or explicit nature of duration processing (Coull and Nobre, 2008).

Based on evidence from neuropsychological and neuroimaging studies, a further distinction has been recently proposed between an Externally-Cued and an Internally-Based timing systems, that may be respectively responsible for the processing of durations when a predictable environmental cue is provided, and when such a cue is lacking (Teghil et al., 2019). This distinction has been supported by meta-analytic evidence, suggesting that two partly overlapping brain networks mediate timing in the two contexts (Teghil et al., 2019). Behavioral evidence in healthy participants is also consistent with the possibility to dissociate, at least partially, these two processes, showing that interval reproduction accuracy in irregular, but not in regular contexts, is predicted by individual differences in interoceptive awareness (Teghil et al., 2020a). Since interoceptive awareness critically depends on the right insular cortex (Craig, 2002; Critchley et al., 2004; Grossi et al., 2014), these findings suggest that the right insula may be especially involved in timing when no structured signal is provided to aid time estimation. Consistently with this hypothesis, individual performance in timing in irregular contexts has been found to predict the strength of the resting-state functional coupling between nodes of a sensorimotor network involving the right insula; furthermore, the strength of the connectivity profiles of the right posterior insula, within a network modulated by individual differences interoceptive awareness, positively correlates with timing performance in irregular but not in regular contexts (Teghil et al., 2020b).

In the present study we aimed to provide neuropsychological evidence for the possibility to dissociate brain correlates of Internally-Based and Externally-Cued timing. Though studies on Parkinson's Disease and cerebellar degeneration patients are consistent with the possibility to distinguish between these two systems (Nichelli et al., 1996; Grube et al., 2010; Harrington et al., 1998a, 1998b; Freeman et al., 1993; Grahn and Brett, 2009), it is not entirely clear whether at least partially separated brain regions are recruited in timing depending on whether any external predictable temporal cue is provided. The interpretation of the neuropsychological data reviewed above is also complicated by relevant differences between studies in stimuli, experimental settings, and in the range of durations tested. To the best of our knowledge, no study to date has tested the effect of the presence vs. absence of external regular cues on duration processing in the same population of patients, assessing the effect of lesion to different brain regions, and using entirely comparable stimuli and procedures.

Here we assessed duration reproduction in regular and irregular contexts in a group of patients with unilateral focal brain lesions, in order to investigate neural substrates of interval reproduction when an external regular cue is provided (regular condition), and when the 
external cue is irregular, and thus uninformative about elapsed time (irregular condition). Moreover, based on previous findings (Teghil et al., 2020a, 2020b), we predicted that a worse performance in the irregular, but not in the regular condition, should be associated with lesions to the right insular cortex, that is pivotal for interoceptive awareness. In the present study, we assessed the reproduction of relatively long suprasecond durations (approximately between 8 and 19 seconds). This choice is in line with previous behavioral and psychophysiological studies, showing a positive association between time processing in this range, and individual differences in autonomic activity and interoceptive processing (Meissner and Wittmann, 2011; Teghil et al., 2020a). Also, neuroimaging studies suggest that processing of durations in this range entails increased activation of the insular cortex (Wittmann et al., 2010, 2011), and is associated with individual variations in the intrinsic connectivity of the right insula (Teghil et al., 2020b).

The relation between behavioral timing performance and lesion data was assessed in two ways: 1) from a topological perspective, using Voxel-Based Lesion-Symptom Mapping (VLSM) (Bates et al., 2003), and 2) from a hodological perspective, using atlas-based tractography (Thiebaut de Schotten et al., 2015; Dalla Barba et al., 2018; Pacella et al., 2019).

\section{Materials and methods}

\subsection{Participants}

19 patients with unilateral focal brain lesions (BD group), admitted to the Neuropsychology Unit of the IRCCS Fondazione Santa Lucia, took part in the study. 5 patients had a left-sided brain lesion, whereas 14 patients had a right-sided brain lesion. Demographical data of the patients are reported in Table 1 . All patients performed within the normal range in standard tests assessing abstract (Raven's Coloured Progressive Matrices, Basso et al., 1987) or verbal reasoning (Verbal Judgment Test, Spinnler and Tognoni, 1987) (two patients showing evidence of reduced visual field were assessed for general reasoning abilities using the Verbal Judgment Test, Spinnler and Tognoni, 1987). All left-hemisphere brain damaged patients showed no evidence of verbal comprehension deficits, as evaluated with the Token Test (Spinnler and Tognoni, 1987) or the
Sentence Comprehension subtest of the ENPA (Capasso and Miceli, 2001). 4 right-hemisphere brain damaged patients showed evidence of unilateral neglect, as assessed with the Letter Cancellation Test, the Line Cancellation Test, the Wundt-Jastrow Area Illusion Test, and the Sentence Reading Test. According to normative data (Pizzamiglio et al., 1989), patients were diagnosed as having extrapersonal neglect if they performed below the cut-off in at least two tests out of four. The screening for verbal comprehension and for neglect symptoms was performed by an expert neuropsychologist, upon admission to the Neuropsychology Unit. Patients' scores on tests assessing verbal comprehension and unilateral neglect are reported in Supplementary materials.

27 healthy controls (CT group), matched with the BD group for age (BD group: $\mathrm{M}=56.842$ years, $\mathrm{SD}=13.696$, CT group: $\mathrm{M}=55.851$ years, $\mathrm{SD}=8.583, t(27.851)=0.279, p=0.782$, two-tailed) and education (BD group: $\mathrm{M}=13.684$ years, $\mathrm{SD}=3.367$, CT group: $\mathrm{M}=12.963$ years, $\mathrm{SD}=3.557, t(44)=0.692, p=0.493$, two-tailed), were also enrolled in the study. The proportion of females and males was not significantly different between the BD and CT groups $(\chi 2=2.477, p=0.116)$. None of the control participants had a previous or current history of neurological or psychiatric disorders, as assessed by an informal interview before the testing session. All control participants were right-handed, and had normal audition and normal or corrected-to-normal vision. All control participants enrolled in the study scored within the normal range in the Raven's Coloured Progressive Matrices (Basso et al., 1987). The study was designed in accordance with the principles of the Declaration of Helsinki and was approved by the ethical committee of the IRCSS Fondazione Santa Lucia, Rome. Informed consent was obtained from all individual participants included in the study.

\subsection{Interval reproduction task}

All patients and controls performed an adapted version of an interval reproduction task extensively described in previous reports from our group (Teghil et al., 2020a, 2020b) (Fig. 1). Participants were asked to reproduce the time interval elapsed between two auditory signals; each trial involved an encoding and a reproduction phase. During the encoding phase, two auditorily presented Start and End signals marked a target duration. Right after the encoding phase, the reproduction phase

Table 1

Demographic data of participants of the brain damaged patients' group.

\begin{tabular}{|c|c|c|c|c|c|c|c|c|}
\hline Participant & Group & Gender & Age (years) & Education (years) & Handed-ness & Etiology & Time from onset (days) $^{c}$ & Abstract/Verbal reasoning \\
\hline Pt 1 & LBD & M & 68 & 8 & $\mathrm{R}$ & $\mathrm{H}$ & 213 & $15 / 36^{\mathrm{a}}$ \\
\hline Pt 2 & LBD & M & 75 & 18 & $\mathrm{R}$ & $\mathrm{H}$ & 33 & $30 / 36^{a}$ \\
\hline Pt 3 & LBD & M & 62 & 13 & $\mathrm{R}$ & I & 47 & $29 / 36^{a}$ \\
\hline Pt 4 & LBD & M & 65 & 18 & $\mathrm{R}$ & I & 53 & $33 / 36^{a}$ \\
\hline Pt 5 & LBD & M & 52 & 13 & $\mathrm{R}$ & I & 80 & $24 / 36^{a}$ \\
\hline Pt 6 & $\mathrm{RBD}(\mathrm{N}-)$ & $\mathrm{F}$ & 24 & 15 & $\mathrm{R}$ & $\mathrm{H}$ & 43 & $36 / 36^{\mathrm{a}}$ \\
\hline Pt 7 & RBD (N-) & $\mathrm{F}$ & 60 & 15 & $\mathrm{R}$ & I & 36 & $19 / 36^{\mathrm{a}}$ \\
\hline Pt 8 & $\mathrm{RBD}(\mathrm{N}-)$ & M & 72 & 8 & $\mathrm{R}$ & I & 38 & $24 / 36^{a}$ \\
\hline Pt 9 & RBD (N-) & $\mathrm{F}$ & 30 & 18 & $\mathrm{R}$ & I & 45 & $26 / 36^{a}$ \\
\hline Pt 10 & RBD (N-) & M & 54 & 11 & $\mathrm{R}$ & I & 29 & $29 / 36^{a}$ \\
\hline Pt 11 & RBD (N-) & $\mathrm{F}$ & 54 & 18 & $\mathrm{R}$ & $\mathrm{H}$ & 38 & $35 / 36^{a}$ \\
\hline Pt 12 & RBD (N-) & $\mathrm{F}$ & 51 & 13 & $\mathrm{R}$ & I & 40 & $34 / 36^{a}$ \\
\hline Pt 13 & RBD (N-) & $\mathrm{F}$ & 44 & 13 & $\mathrm{R}$ & $\mathrm{H}$ & 60 & $19 / 36^{\mathrm{a}}$ \\
\hline Pt 14 & RBD (N-) & M & 46 & 18 & $\mathrm{R}$ & I & 32 & $36 / 36^{a}$ \\
\hline Pt 15 & $\mathrm{RBD}(\mathrm{N}-)$ & $\mathrm{F}$ & 65 & 13 & $\mathrm{R}$ & I & 32 & $23 / 36^{a}$ \\
\hline Pt 16 & $\mathrm{RBD}(\mathrm{N}+)$ & $\mathrm{F}$ & 74 & 13 & $\mathrm{R}$ & I & 104 & $56 / 60^{b}$ \\
\hline Pt 17 & $\mathrm{RBD}(\mathrm{N}+)$ & M & 59 & 8 & $\mathrm{R}$ & $\mathrm{T}$ & 50 & $20 / 36^{a}$ \\
\hline Pt 18 & $\mathrm{RBD}(\mathrm{N}+)$ & M & 61 & 14 & $\mathrm{R}$ & I & 44 & $45 / 60^{b}$ \\
\hline Pt 19 & $\mathrm{RBD}(\mathrm{N}+)$ & $\mathrm{F}$ & 64 & 13 & $\mathrm{R}$ & I & 85 & $17 / 36^{\mathrm{a}}$ \\
\hline
\end{tabular}

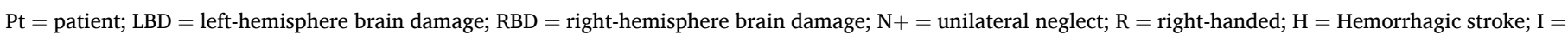
Ischemic stroke; $\mathrm{T}=$ Tumor excision.

a Abstract reasoning (Raven's Coloured Progressive Matrices, Basso et al., 1987).

b Verbal reasoning (Verbal Judgment Test, Spinnler and Tognoni, 1987). Raw scores are reported for the neuropsychological tests. All scores were within the normal range after correction for age and education.

c Time elapsed between the brain lesion and the experimental testing session. 


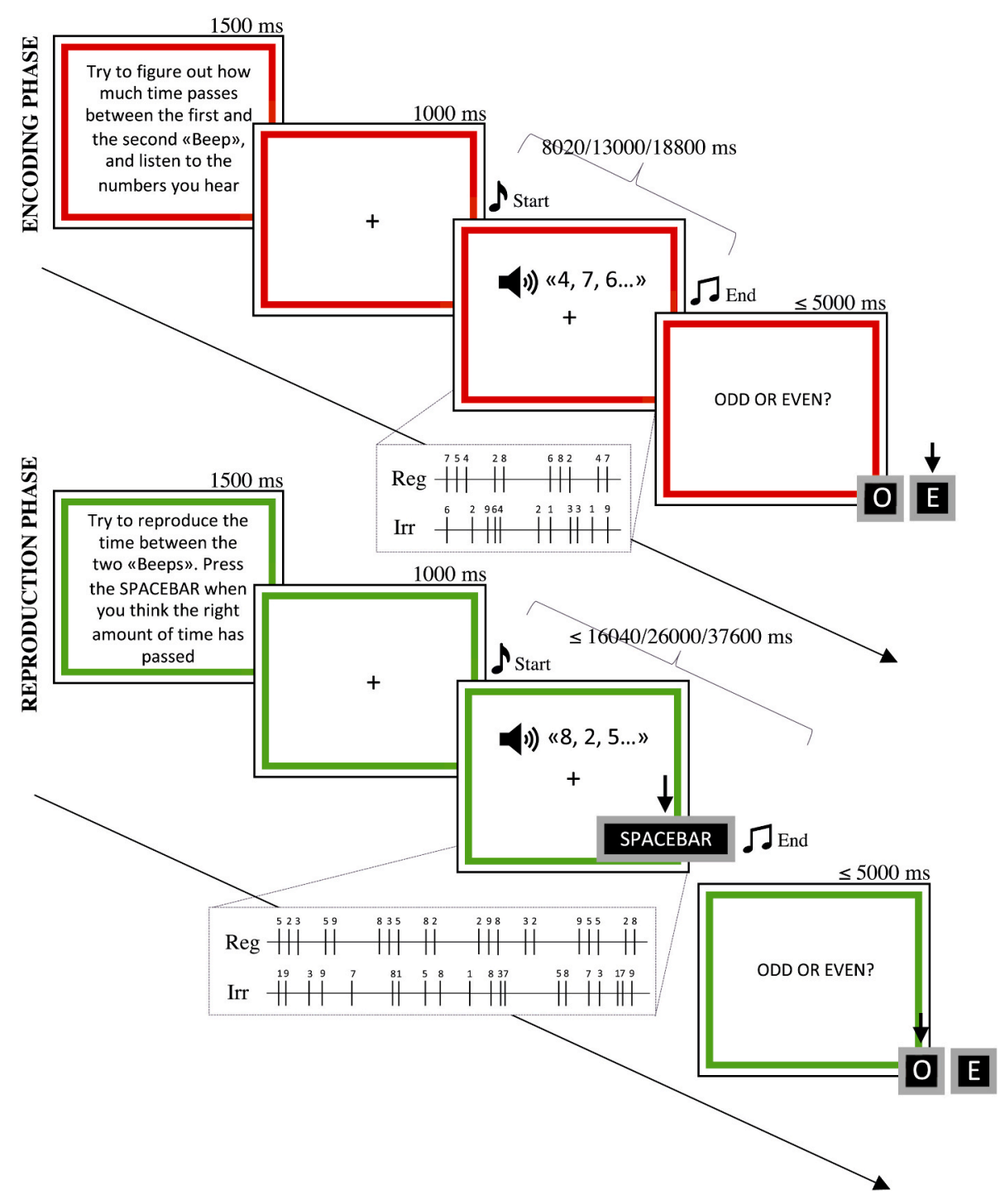

Fig. 1. A trial of the interval reproduction task. The encoding and the reproduction phase were respectively marked by a red and a green frame (adapted from Teghil et al., 2020a). Please note that here the secondary memory task required to report whether the last presented number of the series was odd or even, at difference with previous studies using the same paradigm (Teghil et al., 2020a, 2020b), in which the secondary memory task required to judge whether or not a number was present in the series. (For interpretation of the references to color in this figure legend, the reader is referred to the Web version of this article.) started: the Start signal was presented again, marking the beginning of the reproduction interval, and participants were instructed to press a key (the spacebar on a laptop) when they thought the same time interval was elapsed as that between the two signals in the encoding phase. The spacebar press caused the delivery of the End signal. Participants were asked not to count, nor to use any other vocal or subvocal rehearsal strategy to estimate time; a secondary memory task was added to further prevent counting. Both during the encoding and the reproduction phase, a series of numbers was delivered acoustically within the Start and End signals. In both phases, after the End signal a question appeared on screen asking whether the last presented number was odd or even, and participants answered pressing one of two keys. In order to reduce the cognitive load due to the dual-task demands of the paradigm, the secondary memory task was modified with respect to our previous studies (Teghil et al., 2020a, 2020b), in which a number appeared on screen and participants were asked to decide whether or not it was present in the series. Two conditions were arranged by varying inter-stimulus interval parameters between acoustically presented numbers: in the Regular condition (Reg), the inter-stimulus intervals determined a regular pattern of numbers presentation. The pattern did not vary from the encoding to the reproduction phase of a single trial, thus providing a cue that participants could exploit to reproduce the target interval. A different regular pattern was used in each trial of the Reg condition. In the Irregular condition (Irr), the inter-stimulus intervals were arranged randomly during both the encoding and the reproduction phase, defining an irregular pattern of presentation of the numbers. A different number of numeric stimuli was presented during the same time-window in the encoding and in the reproduction phase of the Irr condition, thus avoiding that participants may exploit information about the "quantity" of numbers presented in the encoding phase to solve the reproduction task. Target durations for the interval reproduction task were 8020 , 13000, $18800 \mathrm{~ms}$. Each number was presented for $400 \mathrm{~ms}$; inter-stimulus intervals between numbers varied from 250 to $1700 \mathrm{~ms}$.

The start of each trial was paced by the experimenter through a spacebar press. 4 trials for each duration were presented within each condition (12 trials for each condition). Reg and Irr trials were arranged in two blocks; trials were randomized within the block. Prior to the reproduction task, participants went into a short familiarization session ( 2 trials for condition), with the same structure of the main task. The presentation order of the regular (Reg) and irregular (Irr) condition was counterbalanced across participants of each group. Stimuli were presented on a laptop using the E-Prime 3.0 software (Psychology Software Tools, Pittsburgh, PA). 


\subsection{Single-case analysis}

As a first step of the lesion-symptom analysis, we assessed whether any participant of the BD group showed evidence of a dissociation between performance in the Reg and Irr condition. This analysis was performed using the DissocsBayes.exe program (Crawford and Garthwaite, 2007). In short, the program tests whether scores of a patient on two tasks are significantly different (lower) from those of a control sample; then, it applies the Bayesian Standardized Difference Test (BSDT) to test the probability that the standardized difference between the patient's scores on the two tasks is an observation from the control population (Crawford and Garthwaite, 2007). According to Crawford and Garthwaite's (2007) Bayesian criteria for classical and strong dissociations, the criteria for a dissociation, putatively classical, are fulfilled when a patient's score on one of the two tasks is low enough that the probability that it is an observation from the control population is $<0.05$ (Crawford and Howell, 1998), and the probability that the standardized difference between the patient's scores on the two tasks is an observation from the control population is $<0.05$ on the BSDT (Crawford and Garthwaite, 2007). The criteria for a strong dissociation are fulfilled when the patient's scores on both tasks are low enough that the probability that they are observations from the control population is $<0.05$ (Crawford and Howell, 1998), and there is also a significant difference on the BSDT between standardized scores on the two tasks (Crawford and Garthwaite, 2007). Analyses were performed on mean accuracy scores in the Reg and Irr conditions (see Section 3.1).

\subsection{Lesion mapping}

Computed Tomography/Magnetic Resonance (CT/MRI) digitalized images were acquired for each patient. Lesions were manually drawn on representative axial slices of a standard MNI152 Colin27 template using MRIcron (available at https://www.nitrc.org/projects/mricron) (Rorden and Brett, 2000), rotating the MNI template from the MNI space to the subject space. The reoriented templates were then taken back to the MNI space using the inverse rotation (Doricchi, 2003). Brain structures highlighted by the VLSM analyses were identified referring to the Rojkova and colleagues' 2016 atlas for white matter structures (Rojkova et al., 2016), and to the automated anatomical atlas (AAL) (Tzourio-Mazoyer et al., 2002) for gray matter structures.

\subsection{Hodological atlas-based lesion-behavior analysis}

We used the Tractotron software (part of the BCBtoolkit, Foulon et al., 2018, http://www.toolkit.bcblab.com) to investigate which tracts were affected by individual lesions. Each lesion was mapped onto tractography reconstructions of white matter pathways obtained from a group of healthy participants (Rojkova et al., 2016). The probability of the tract to be disconnected was analyzed as a measure of disconnection severity (Thiebaut de Schotten et al., 2014). For each patient, a tract was considered to be disconnected when a voxel belonging to that tract fell within the individual lesion with above-chance probability $(p>0.5)$. Only tracts that were disconnected in at least $20 \%$ of patients were considered in further analyses.

\section{Results}

\subsection{Interval reproduction task}

Statistical analyses were performed using SPSS (IBM SPSS Statistics 20). A mean accuracy score in the Reg and Irr condition was computed for each participant of the BD and CT groups, using the formula 1-| (Target duration - Reproduced duration)/Target duration|. Trials in which accuracy was below 2 SDs from the participant's mean accuracy in the specific condition were excluded from further analyses; this led to the exclusion of $2.6 \%$ of trials for the BD group and of $4.16 \%$ of trials in the CT group.

First, in order to assess whether the different target durations in the interval reproduction task were discriminated effectively by participants, a $2 \times 3 \times 2$ mixed-factorial ANOVA was performed on mean reproduced duration in the task, with Condition (Reg, Irr) and Target duration $(8020,13000,18800 \mathrm{~ms})$ as repeated measures, and Group $(\mathrm{BD}, \mathrm{CT})$ as between-subjects factor. When a departure from sphericity was detected, a Greenhouse-Geisser's correction was adopted. There was no significant effect of Condition $\left(F(1,44)=0.001, p=0.978, \eta_{\mathrm{p}}^{2}<\right.$ $0.001)$, whereas the effect of Target duration was significant $(F(1.279$, $56.268)=253.48, p<0.001, \eta_{p}^{2}=0.852$ ), all comparisons between mean reproduced duration for each target duration being also significant $(p<0.001$, Bonferroni corrected). The main effect of Group was not significant $\left(F(1,44)=0.477, p=0.493, \eta_{p}^{2}=0.011\right)$, neither was any interaction effect (Condition x Group: $F(1,44)=0.201, p=0.656, \eta^{2}=$ 0.005; Target duration x Group: $F(1.279,56.268)=0.155, p=0.757$, $\eta_{p}^{2}=0.004$; Condition $\mathrm{x}$ Target duration: $F(1.537,67.61)=0.79, p=$ $0.428, \eta_{\mathrm{p}}^{2}=0.018$; Condition $\mathrm{x}$ Target duration $\mathrm{x}$ Group: $F(1.537$, $67.61)=0.168, p=0.789, \eta_{p}^{2}=0.004$ ) (Fig. 2a). For exploratory purposes, $2 \times 3 \times 3$ mixed-factorial ANOVAs were also performed to compare 1) the mean reproduced durations, and 2) the ratio of the reproduced-to-target duration, in the two conditions of the task, between patients with lesion to the right hemisphere, patients with lesion to the left hemisphere, and controls. Results of these analyses are provided in Supplementary materials.

A $2 \times 2$ mixed-factorial ANOVA was performed to compare accuracy scores in the two Conditions (Reg, Irr) between the two groups (BD, CT). Since the results of the ANOVA on mean reproduced durations showed that the three target durations were correctly discriminated in both groups, only mean accuracy in the Reg and Irr conditions was considered in this and in further analyses. There was no main effect of Condition ( $F$ $\left.(1,44)=3.357, p=0.074, \eta^{2}=0.071\right)$, whereas the effect of Group was significant $\left(F(1,44)=4.55, p=0.039, \eta_{p}^{2}=0.094\right.$ ) (Fig. 2b). The Condition $\times$ Group interaction was not significant $(F(1,44)=0.179, p$ $\left.=0.674, \eta_{\mathrm{p}}^{2}=0.004\right)$. An additional exploratory ANOVA comparing accuracy scores in the two Conditions between controls, and patients with lesion to the left and right hemisphere, also highlighted only a significant effect of Group (results of this analysis are provided in Supplementary materials).

\subsection{Single-case analysis}

One patient (Pt 8) fulfilled the criteria for a dissociation, putatively classical (Crawford and Garthwaite, 2007). Pt 8's mean accuracy was similar to that of controls in the Reg condition $(t(26)=-0.223, p=$ 0.413 , one-tailed), but not in the Irr condition $(t(26)=-3.101, p=$
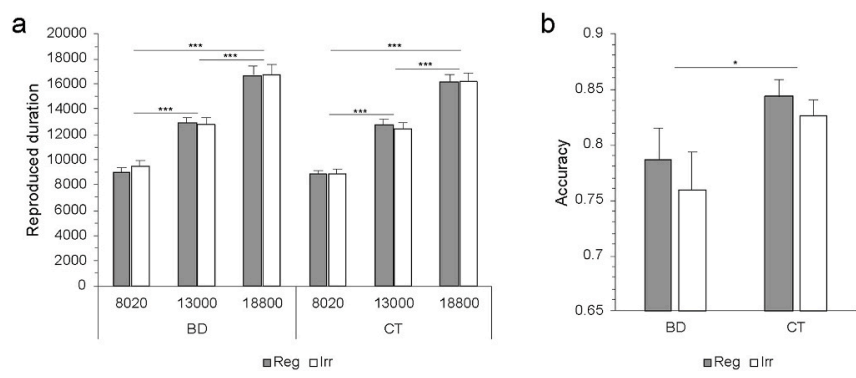

Fig. 2. Interval reproduction task a) Mean reproduced duration in the Regular (Reg) and Irregular (Irr) condition for each target duration (8020, 13000, $18800 \mathrm{~ms}$ ), in the brain damaged (BD) and control (CT) groups. b) Mean accuracy in the Regular (Reg) and Irregular (Irr) condition in the brain damaged (BD) and control (CT) groups. Bars represent standard errors. Significant differences are marked with asterisks. ${ }^{*} p<0.05 * * * p<0.001$. 
0.002 , one-tailed), and the difference between the patient's standardized scores in the two conditions was statistically significant on the BSDT [percentage of control population with a more extreme discrepancy in the same direction as patient: Bayesian point estimate $(+95 \%$ CI) $=0.750 \%(95 \% \mathrm{CI}=0.0096 \%-3.8845 \%)$ ]. Lesion of Pt 8 involved the white matter of fronto-temporal and parietal regions in the right hemisphere, extending to the caudate nucleus and to the posterior insular cortex, at the boundary with the parietal and temporal lobe. CT scan of Pt 8 is shown in Fig. 3.

\subsection{Voxel-based lesion-symptom mapping (VLSM) analysis}

Lesions overlap of participants of the BD group is shown in Fig. 4. A Voxel-Based Lesion-Symptom Mapping (VLSM) analysis (Bates et al., 2003) was performed using the NiiStat package (http://www.nitrc.or $\mathrm{g} /$ projects/niistat/). In VLSM, the relation between behavioral scores and lesion information is assessed voxel-wise, comparing for each voxel behavioral scores of patients with and without lesions involving that voxel (Bates et al., 2003). Mean accuracy of patients in the Reg and the Irr condition were entered in the analysis. $T$-tests were performed to test for significant difference at each voxel, testing only voxels damaged in at least 5 patients. Lesion volume was entered as a covariate of no interest, regressing out its effect on the behavioral scores. Permutation thresholding was used to correct for multiple comparisons, using 5000 permutations, with alpha level set at $p<0.05$, one tailed.

VLSM results showed that scores in the Reg condition were associated with lesions to clusters of voxels in the white matter of the right hemisphere, involving the anterior thalamic projection, the corpus callosum, the cortico-spinal and fronto-striatal tracts, and the superior longitudinal fasciculus II and III, partially extending to the frontal commissural tract and to the arcuate long segment (Fig. 5).

Lower scores in the Irr condition were also associated with lesions in clusters of voxels in the right hemisphere, involving the anterior thalamic projection, the arcuate anterior and long segments, the corpus callosum, the frontal commissural tract, the cortico-spinal tract, the fronto-striatal tract, the fronto-insular tract 5 , the superior longitudinal fasciculus II and III, and extending to the frontal aslant tract, the hand inferior $\mathrm{U}$ tract, the superior longitudinal fasciculus I, and to the insularopercular cortex (Fig. 6).

\subsection{Relation between probability of disconnection and behavioral measures}

Pearson's one-tailed partial correlations were performed between the probability of disconnection of the white matter tracts, and patients' scores in the Reg and Irr conditions, entering the volume of individual lesions as a covariate of no interest. Accuracy scores in both the Reg and the Irr conditions were significantly inversely correlated with the probability of disconnection of the right superior longitudinal fasciculus I (Reg: $r=0.337, p=0.026$, Irr: $r=-0.455, p=0.029$ ) (Fig. 7a), the right superior longitudinal fasciculus II (Reg: $r=-0.574, p=0.006$, Irr: $r=-0.516, p=0.014$ ) (Fig. 7b) and the right frontal superior longitudinal tract (Reg: $r=-0.587, p=0.005$, Irr: $r=-0.506, p=0.016$ ) (Fig. 7c).

\section{Discussion}

In the present study we aimed to investigate neural substrates of duration processing in different sensory contexts. In more detail, we assessed whether at least partially different brain regions support interval reproduction in contexts that allow to estimate time relying on a predictable cue, compared to when the perception of elapsed time is less likely to be informed by external referents. Results of previous behavioral (Teghil et al., 2020a) and neuroimaging (Teghil et al., 2020b) studies provided early support to the hypothesis that interval timing more likely relies on the integration of endogenous inputs when the external environment does not allow to predict the duration of events. Thus, based on evidence on the role of insula in interoception (Critchley et al., 2004; Craig, 2002; Grossi et al., 2014) and time perception (Wittmann et al., 2010, 2011; Meissner and Wittmann, 2011; Monfort et al., 2014; Teghil et al., 2020b), as a further and more specific aim we tested the hypothesis that duration processing in irregular, unpredictable contexts specifically relies on the right insular cortex.

To these aims we used VLSM, a data-driven analytic technique that allows to assess the relation between continuous behavioral scores and lesion information on a voxel-by-voxel basis (Bates et al., 2003). In other words, rather than relying on a priori group divisions - such as the presence/absence of a deficit - VLSM treats behavioral scores in a continuous manner. This technique thus overcomes the intrinsic limitations of grouping patients according to lesion site or to their performance in behavioral tasks, such as the impossibility to draw conclusions about the contribution of other regions to the investigated process, and the loss of information related to more fine-grained performance differences (Bates et al., 2003; Coslett et al., 2009). Furthermore, we investigated brain correlates of duration processing in regular vs. irregular contexts from a hodological perspective, assessing the relation between timing accuracy in the two contexts and the probability of disconnection of specific white matter tracts.

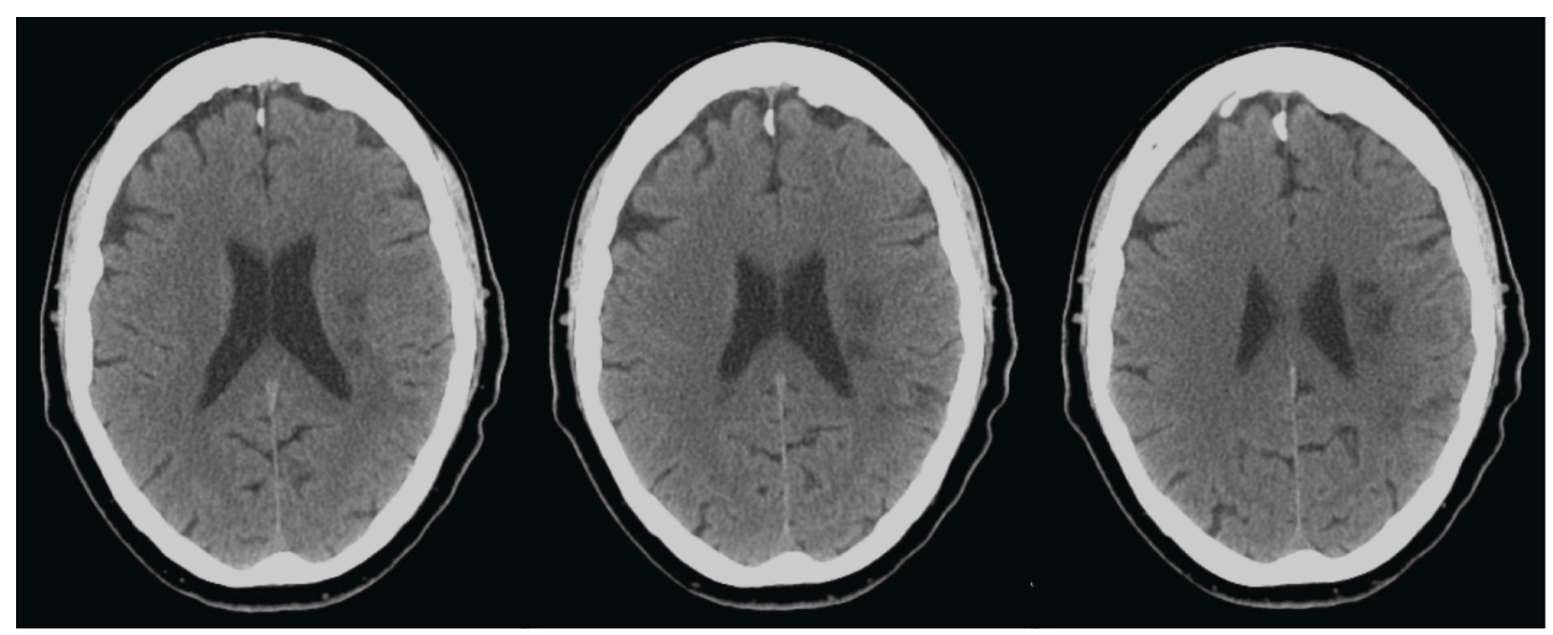

Fig. 3. CT scan of Pt 8 (right on the right side). 

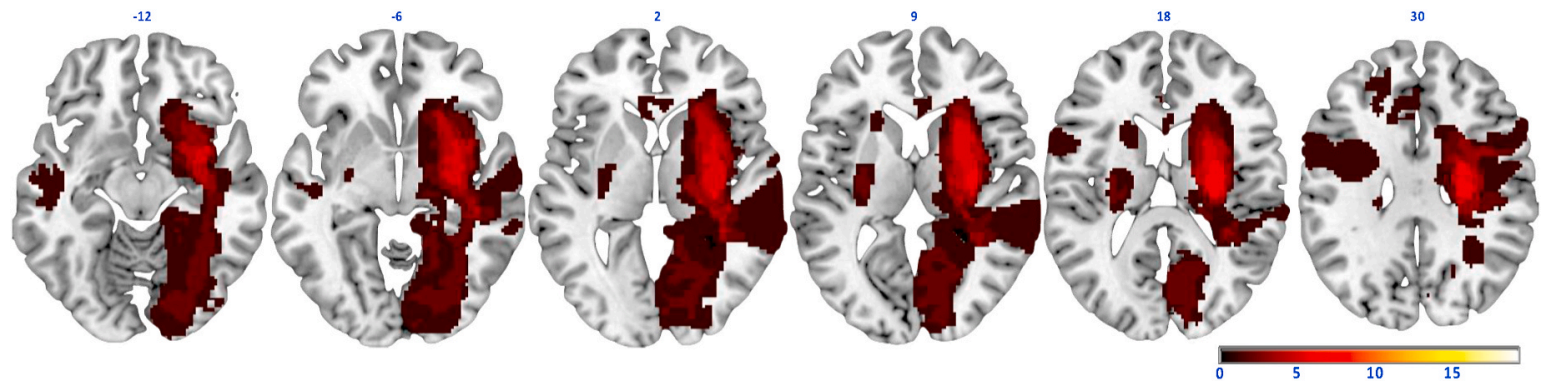

Fig. 4. Lesion overlap of participants of the BD group $(n=19)$, displayed for visualization purposes. The color bar represents the number of patients with lesion involving each brain voxel. (For interpretation of the references to color in this figure legend, the reader is referred to the Web version of this article.)

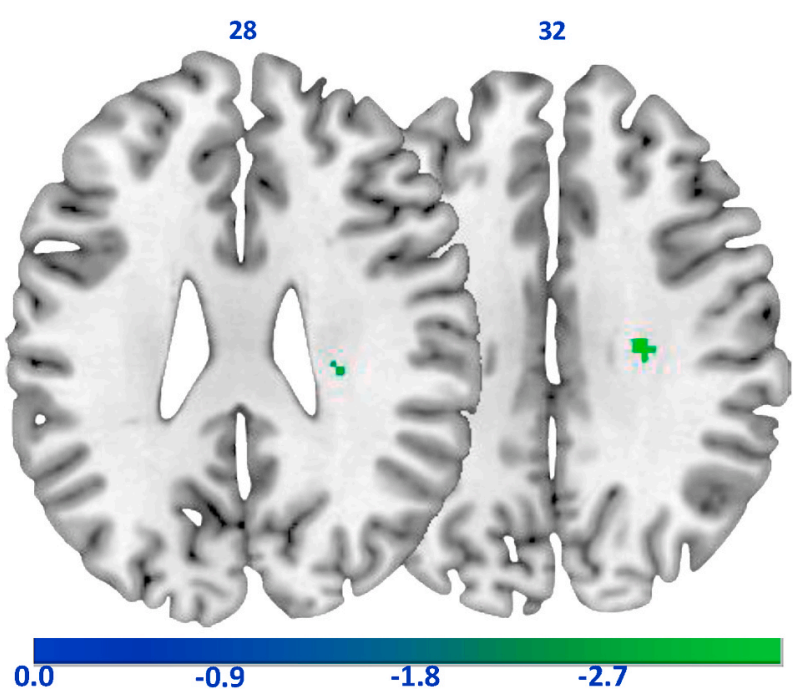

Fig. 5. Z statistic, corresponding to the t-test, comparing scores in the Regular condition between patients with or without lesions involving each voxel. Only voxels surviving a permutation thresholding of $p<0.05$ one-tailed are shown.

VLSM analyses showed that lower accuracy in both the Regular and in the Irregular conditions was mainly associated with right-hemisphere white matter lesions. However, scores in the two conditions were associated with lesions to partly separable clusters of voxels. Indeed, though lower performances in both measures were associated with lesions involving the anterior thalamic projection, the cortico-spinal tract, the fronto-striatal tract, the corpus callosum and the superior longitudinal fasciculi II and III, clusters of voxels related to low performance in the Regular condition extended more medially, whereas lower performance in the Irregular condition was also related to lesions involving the arcuate anterior and long segments, the frontal commissural tract, the fronto-insular tract 5 and the insular cortex (Figs. 5 and 6). This latter result is thus consistent with previous evidence that the right insular cortex may specifically play a role in processing duration information independently from the surrounding context (Teghil et al., 2019, 2020a, 2020b).

Results of single-case analyses also suggest a classical dissociation between duration reproduction in regular and irregular contexts. One patient, indeed, performed significantly worse than controls in the irregular, but not in the regular condition, and fulfilled the criteria for a dissociation, putatively classical (Crawford and Garthwaite, 2007). Thus, the patient's performance was comparable to that of controls when an external regular cue was available, but not when the external cue was provided at non-predictable timepoints, not being informative about elapsed time. This suggests that the patient was indeed able to exploit information provided by the regular cue to improve duration reproduction, whereas hisperformance dropped substantially when durations were to be reproduced entirely based on a subjective estimate of elapsed time. Interestingly, this pattern of performance was associated with a lesion involving the white matter of the frontal and temporal lobe, extending to the posterior portion of the insula and of the parietal lobe. This finding is thus consistent with the hypothesis of an

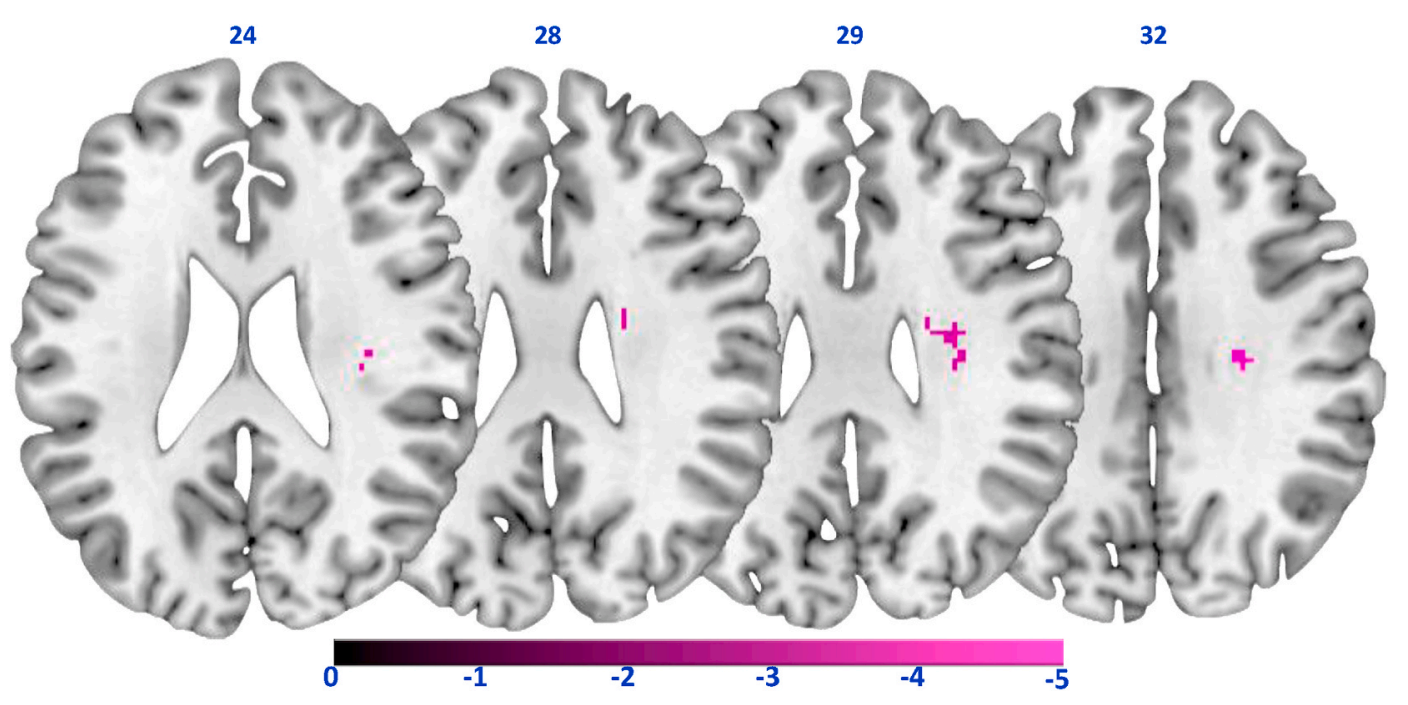

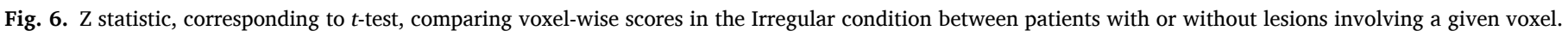
Only voxels surviving a permutation thresholding of $p<0.05$ one-tailed are shown. 

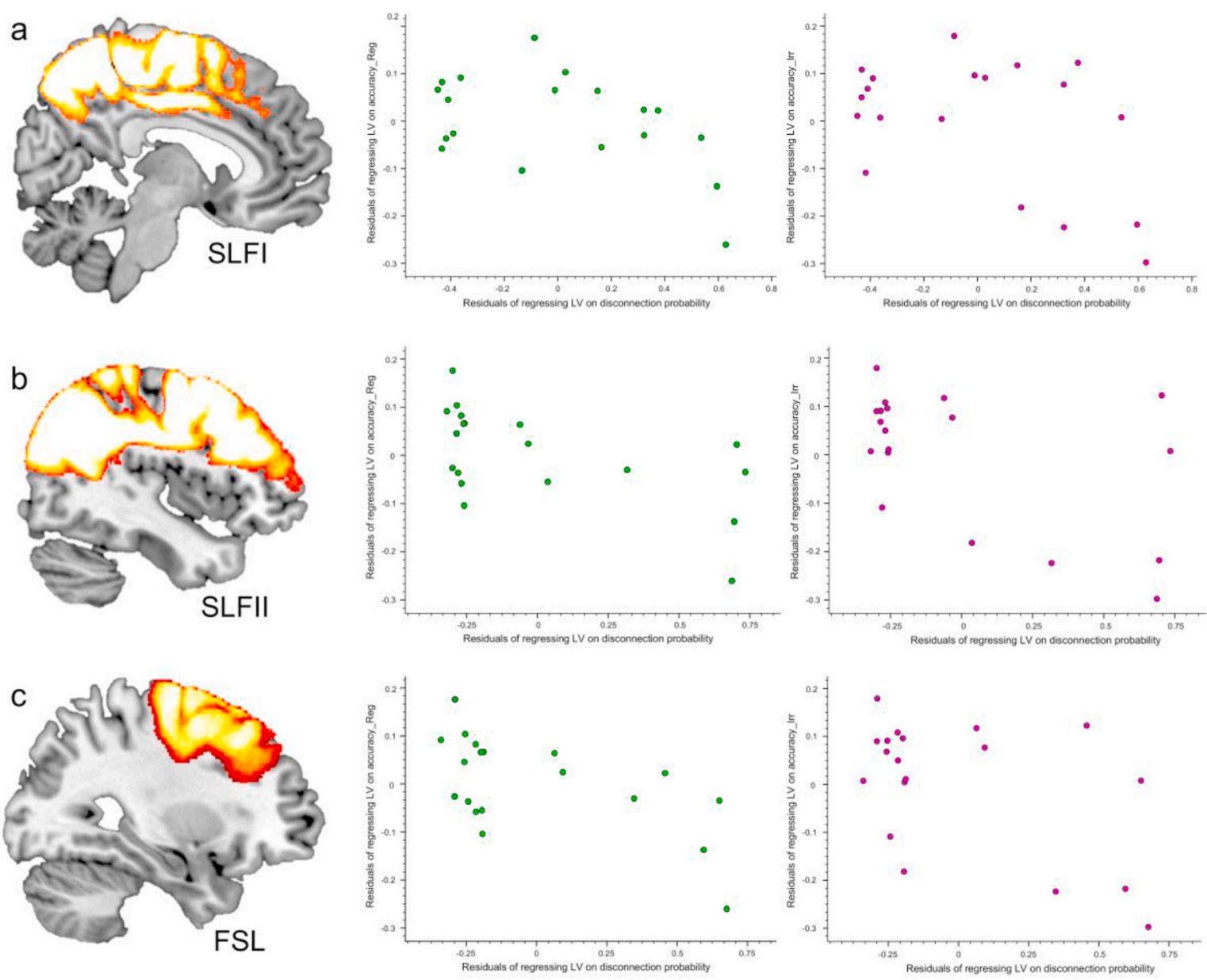

Fig. 7. Scatterplots showing the relation between the probability of disconnection (x axis) and the behavioral measures (y axis), after regressing out the effect of individual lesion volume (LV). Relation between residuals of the regressions of LV on the probability of disconnection of a) the right superior longitudinal fasciculus I (SLFI), b) the right superior longitudinal fasciculus II (SLFII), c) the right frontal superior longitudinal tract, and residuals of the regressions of LV on accuracy in the Regular (Reg) (left side) and Irregular (Irr) condition (right side).

involvement of right insular regions and surrounding white matter in the perception of durations when no external temporal cue is provided. It is important to point out that the single-case approach has some intrinsic limitations: indeed, it allows drawing only limited anatomical inference, mainly due to the difficulties in generalizing the results (Robertson et al., 1993; Rorden et al., 2007). Nonetheless, present single-case results converge with those of the VLSM analysis in suggesting the involvement of the right insula in timing in irregular contexts.

As noted above, VLSM analyses linked lower performances in both the regular and irregular condition to lesions to clusters of voxels largely involving the white matter. Results of the hodological symptom-lesion analyses are thus of particular interest. Lower accuracy scores in both the regular and the irregular condition were found to correlate negatively with the probability of disconnection of the superior (SLF I) and middle (SLF II) branches of the right superior longitudinal fasciculus, along with the probability of disconnection of the right frontal superior longitudinal tract. The superior longitudinal fasciculus connects different frontal and parietal regions; specifically, the SLF I connects the superior parietal lobule and the precuneus to the superior frontal gyrus, including the supplementary motor area (SMA), the frontal eye fields, and dorsolateral and orbitofrontal regions, whereas the SLF II connects the angular gyrus to the middle frontal gyrus and the dorsolateral prefrontal cortex (Kamali et al., 2014; Rojkova et al., 2016). The frontal superior longitudinal tract, instead, includes long fibers connecting the superior frontal gyrus with the precentral gyrus, as well as short fibers mediating local connections between the superior and middle frontal gyri (Catani et al., 2012; Rojkova et al., 2016). It is worth noting that, despite the long-established role of the SMA in motor functions, a consistent body of literature points to a pivotal role of this region in timing processes across different contexts and conditions, independently from the motor or perceptual nature of the task (see Wiener et al., 2010a; Schwartze et al., 2012; Teghil et al., 2019; Nani et al., 2019, for reviews). Present findings expand upon previous results, suggesting that the SMA may be a key node of a network supporting the representation of duration information both relative to a regular sensory signal, and independently from the external context. Interestingly, this hypothesis is consistent with recent findings from single-cell recording in monkeys. SMA neurons, indeed, exhibit burst onsets that reflect the duration of intervals defining an extinguished isochronous visual rhythm; burst amplitude, however, increases as a function of total elapsed time (Cadena-Valencia et al., 2018), suggesting that neural activity in the SMA may encode both regular patterns, and duration information per se.

The contribution of prefrontal regions to timing has also been highlighted by a number of studies. As reviewed in the Introduction, investigations on brain damaged patients consistently report timing deficits following prefrontal (Harrington et al., 1998b; Koch et al., 2002; Mangels et al., 1998) but also orbitofrontal cortex lesions (Berlin et al., 2004). A role of the right dorsolateral prefrontal cortex in timing has 
also been suggested by neuroimaging studies using different paradigms (Lewis and Miall, 2002; Macar et al., 2002; Jahanshahi et al., 2006; Pouthas et al., 2005; Apaydin et al., 2018; Kale et al., 2019), and rTMS on the right dorsolateral prefrontal cortex has been found to alter duration reproduction of supresecond stimuli (Koch et al., 2003; Jones et al., 2004). The right middle frontal gyrus has also been implied in timing, particularly for suprasecond durations (Wiener et al., 2010a; Gooch et al., 2011). The involvement of prefrontal regions in timing tasks has often been interpreted in terms of working memory demands, especially for timing of suprasecond durations (Harrington et al., 1998b; Mangels et al., 1998; Jones et al., 2004; Lewis and Miall, 2006; Coull et al., 2011). Others, however, have proposed a more "core" role for prefrontal regions in timing processes (Koch et al., 2002; Wiener et al., 2010a). It is worth noting that, though most timing tasks involve different executive resources at least to some degree (Odgen et al., 2014), duration reproduction is particularly dependent on working memory (Perbal et al., 2002; Baudoin et al., 2006; Droit-Volet et al., 2015; Mioni et al., 2013). Our findings of a correlation between reduced interval reproduction accuracy and disconnection of prefrontal regions are thus compatible both with general and specific accounts of the contribution of these areas to timing. However, assuming that a working memory impairment would generally affect duration reproduction of long durations, present findings could likely reflect the strong working memory demands imposed by both the regular and irregular condition.

The relation between disconnection of fibers targeting parietal regions and lower timing performance is also consistent with evidence from neuropsychological studies. The superior parietal lobule, specifically, has been previously implicated in interval reproduction in humans (Coslett et al., 2009), and lesions to the right supramarginal and angular gyrus have also been linked to impaired interval discrimination in brain damaged patients (Harrington et al., 1998b; Danckert et al., 2007). In line with these findings, disruption of activity in the right inferior parietal cortex alters duration discrimination in healthy participants (Alexander et al., 2005; Bueti et al., 2008a Wiener et al., 2010b, 2012). Activation of right parietal regions during timing tasks has also been reported by different fMRI studies, involving duration estimation and reproduction tasks (Lewis and Miall, 2003b; Bueti et al., 2008a, 2008b; Dormal et al., 2012; Oliveri et al., 2009; Aso et al., 2010) but also the extraction of duration information from the structure of the external environment (Li et al., 2015; Apaydın et al., 2018). Our results suggest that the connections of parietal to premotor and prefrontal regions may be involved in duration reproduction in both Internally-Based and Externally-Cued contexts, consistently with evidence that neurons in the lateral intraparietal area encode the probability that an external event will occur (Janssen and Shadlen, 2005), as well as elapsing time during the encoding and reproduction phase of a duration reproduction task (Jazayeri and Shadlen, 2015). These results are thus consistent with accounts suggesting that the right parietal cortex may be a pivotal integration and/or representational hub for temporal processing (Walsh, 2003; Battelli et al., 2007, 2008; Bueti et al., 2008b; Oliveri et al., 2009).

The right precentral gyrus has also been implied in time perception processes; the contribution of this region, however, is presently unclear. Interestingly, lesion to the tight precentral gyrus was associated with the disruption of duration discrimination of both sub- and suprasecond stimuli in the only other previous VLSM study on interval timing (Gooch et al., 2011). A recent Activation Likelihood Estimation and modeled alteration meta-analysis has also reported activation likelihood for the right precentral gyrus in suprasecond non-motor tasks (Nani et al., 2019). Activation of the precentral gyrus in fMRI studies on timing has sometimes been interpreted as reflecting subvocal counting strategies (Hinton et al., 2004; Wiener et al., 2010a; Nani et al., 2019); it is worth noting, however, that this interpretation is usually related to activation of the left rather than right precentral gyrus. Also, the right precentral gyrus has been reported to show increased functional connectivity with the right intraparietal sulcus during an interval discrimination task (Dormal et al., 2012) and to be activated during the reproduction of suprasecond durations (Wittmann et al., 2010a). Both these studies controlled for the use of counting strategies (Dormal et al., 2012; Wittmann et al., 2010a), as in the case of the present study. Our findings thus point to a contribution of intra-frontal connections between the precentral gyrus and the prefrontal cortex in timing that goes beyond verbal rehearsal; however, further studies will be needed to uncover the precise contribution of precentral regions to duration processing.

Present results are overall consistent with previously discussed neuropsychological studies, as well as with neuroimaging literature (see Wiener et al., 2010a; Nani et al., 2019 for meta-analytic reviews), in supporting a pivotal role of premotor, prefrontal and parietal cortex in timing, particularly for suprasecond tasks in which durations are not defined by movements (Lewis and Miall, 2003a).

Here we did not highlight the involvement of a number of structures consistently implicated in time perception by previous studies. Concerning the basal ganglia, the reason of the lack of a consistent relation between lower performance in our task and lesion to, or disconnection of, these regions, is unclear. A possible explanation is related to the nature of the task, that involved suprasecond - and relatively long durations (Lewis and Miall, 2003a; Wiener et al., 2010a, 2011b). The role of basal ganglia in timing of multisecond durations in different contexts, however, deserves further investigation.

We also did not find evidence for cerebellar or hippocampal involvement in our study. These latter results, however, deserve caution, since lesions involved the right peri-sylvian region in the large majority of patients, thus preventing us from having enough statistical power to detect effects in the cerebellum or hippocampus. Furthermore, here we did not highlight any relation between timing in regular and irregular contexts and damage to the left hemisphere. Interestingly, this finding is in line with the results of explorative analyses (see Supplementary materials) comparing accuracy scores in the two conditions of the task between controls, RBD and LBD patients, and showing that the LBD group performed similarly to controls in both conditions. It is worth noting, however, that an important limitation of the present study is that only five LBD patients were included; thus, a lack of statistical power may have prevented us from detecting effects in the in the left hemisphere. Since we aimed to relate structural damage and behavioral measures of timing across the whole brain, and time perception relies on network interactions between multiple brain regions in both hemispheres (Nani et al., 2019; Teghil et al., 2019; Binetti et al., 2020), we choose to include both right and left brain damaged patients, although the two groups were not completely balanced. Present results, however, do not allow to draw definite conclusions on the role of left-sided brain regions in duration reproduction in regular and irregular contexts. Future studies are needed, involving a larger number of patients with lesions to the left hemisphere, to properly highlight the possible differential contribution of the left and right hemispheres to timing in regular and irregular contexts, within large-scale brain networks supporting time processing. Finally, a main limitation of the present study is that sample size was relatively small for the VLSM and tractography analyses; a replication of results in larger samples of brain damaged patients is thus needed to draw conclusive inferences.

Overall, present findings are consistent with previous meta-analytic evidence (Teghil et al., 2019), in showing that the dissociation between internally-based and externally-cued timing processes is not complete. Indeed, worse performance in both conditions of our task was found to be associated with damage to a common suprasecond timing network. Nonetheless, in accordance with findings in healthy individuals (Teghil et al., 2020a, 2020b), we showed that a worse performance in reproducing durations in the irregular condition was selectively related to lesions to the right insular cortex, consistently with the hypothesis that the integration of psychophysiological changes may be particularly relevant when tracking time without the aid of external cues. Results of the present study further expand previous evidence, suggesting that internally-based and externally-cued timing processes recruit partially dissociable brain regions, but also that the disruption of interregional 
brain connections pivotal to the specific task and range of durations probed affects performance in both domains.

More broadly, our findings also support a key role of network connections in timing, consistently with the proposals that timing crucially relies on distributed networks of interconnected brain regions, rather than on a single structure with central time-keeping functions (Koch et al., 2009; Bueti, 2011; Wiener et al., 2011a; Üstü;n et al., 2017; Paton and Buonomano, 2018). Interestingly, although present results suggest that damage to white matter tracts connecting these regions may be related to less accurate timing performance, a contribution of individual differences in patterns of intrinsic functional connectivity to timing has also been recently highlighted (Teghil et al., 2020b). Thus, future lesion-behavior mapping studies of interval timing would benefit from using multimodal imaging protocols, allowing to assess concurrently both structural and functional alterations, including the possible distal disfunctions due to diaschisis phenomena (Carrera and Tononi, 2014; Grefkes and Fink, 2014).

\section{Funding}

This work was partially supported by funding from the Italian Ministry of Health to IRCCS Fondazione Santa Lucia (Ricerca Corrente) and by a fellowship from the PhD Program in Behavioral Neuroscience of "Sapienza University of Rome" to AT.

\section{CRediT authorship contribution statement}

Alice Teghil: Conceptualization, Methodology, Formal analysis, Investigation, Writing - original draft, Writing - review \& editing. Antonella Di Vita: Conceptualization, Methodology, Writing - review \& editing. Veronica Pietranelli: Investigation, Writing - review \& editing. Alessandro Matano: Methodology, Investigation, Writing - review \& editing. Maddalena Boccia: Conceptualization, Methodology, Formal analysis, Supervision, Writing - review \& editing.

\section{Declaration of competing interest}

None.

\section{Appendix A. Supplementary data}

Supplementary data to this article can be found online at https://doi. org/10.1016/j.neuropsychologia.2020.107577.

\section{References}

Alexander, I., Cowey, A., Walsh, V., 2005. The right parietal cortex and time perception: back to Critchley and the Zeitraffer phenomenon. Cogn. Neuropsychol. 22, 306-315. https://doi.org/10.1080/02643290442000356.

Apaydın, N., Ustun, S., Kale, E.H., Celikag, I.., Ozguven, H.D., Baskak, B., Cicek, M., 2018. Neural mechanisms underlying time perception and reward anticipation. Front. Hum. Neurosci. 12, 115. https://doi.org/10.3389/fnhum.2018.00115.

Aso, K., Hanakawa, T., Aso, T., Fukuyama, H., 2010. Cerebro-cerebellar interactions underlying temporal information processing. J. Cognit. Neurosci. 22, 2913-2925.

Basso, A., Capitani, E., Laiacona, M., 1987. Raven's Colored Progressive Matrices: normative values on 305 adults normal controls. Funct. Neurol. 2, 189-194.

Bates, E., Wilson, S.M., Saygin, A.P., Dick, F., Sereno, M.I., Knight, R.T., Dronkers, N.F., 2003. Voxel-based lesion-symptom mapping. Nat. Neurosci. 6, 448-450.

Battelli, L., Pascual-Leone, A., Cavanagh, P., 2007. The' when' pathway of the right parietal lobe. Trends Cognit. Sci. 11, 204-210. https://doi.org/10.1016/j. tics.2007.03.001.

Battelli, L., Walsh, V., Pascual-Leone, A., Cavanagh, P., 2008. The 'when' parietal pathway explored by lesion studies. Curr. Opin. Neurobiol. 18, 120-126. https://doi. org/10.1016/j.conb.2008.08.004.

Baudouin, A., Vanneste, S., Isingrini, M., Pouthas, V., 2006. Differential involvement of internal clock and working memory in the production and reproduction of duration: a study on older adults. Acta Psychol. 121, 285-296. https://doi.org/10.1016/j. actpsy.2005.07.004.

Berlin, H.A., Rolls, E.T., Kischka, U., 2004. Impulsivity, time perception, emotion and reinforcement sensitivity in patients with orbitofrontal cortex lesions. Brain $127(\mathrm{Pt}$ 5), 1108-1126.
Binetti, N., Tomassini, A., Friston, K., Bestmann, S., 2020. Uncoupling sensation and perception in human time processing. J. Cognit. Neurosci. 32, 1369-1380.

Binkofski, F., Block, R.A., 1996. Accelerated time experience after left frontal cortex lesion. Neurocase 2 (6), 485-493. https://doi.org/10.1080/13554799608402424.

Bueti, D., 2011. The sensory representation of time. Front. Integr. Neurosci. 5, 34 https://doi.org/10.3389/fnint.2011.00034.

Bueti, D., Bahrami, B., Walsh, V., 2008a. Sensory and association cortex in time perception. J. Cognit. Neurosci. 20, 1054-1062.

Bueti, D., Walsh, V., Frith, C., Rees, G., 2008b. Different brain circuits underlie motor and perceptual representations of temporal intervals. J. Cognit. Neurosci. 20, 204-214.

Buhusi, C.V., Meck, W.H., 2005. What makes us tick? Functional and neural mechanisms of interval timing. Nat. Rev. Neurosci. 6, 755-765.

Cadena-Valencia, J., Garcia-Garibay, O., Merchant, H., Jazayeri, M., de Lafuente, V., 2018. Entrainment and maintenance of an internal metronome in supplementary motor area. Elife 7, e38983. https://doi.org/10.7554/eLife.38983.

Capasso, R., Miceli, G., 2001. Esame Neuropsicologico Per l'Afasia. E. N. P. A. Metodologie Riabilitative in Logopedia, vol. 4. Springer Verlag, Milano.

Carrera, E., Tononi, G., 2014. Diaschisis: past, present, future. Brain 137 (Pt 9), 2408-2422. https://doi.org/10.1093/brain/awu101.

Catani, M., Dell'acqua, F., Vergani, F., Malik, F., Hodge, H., Roy, P., Valabregue, R., Thiebaut de Schotten, M., 2012. Short frontal lobe connections of the human brain. Cortex 48, 273-291. https://doi.org/10.1016/j.cortex.2011.12.001.

Coslett, H.B., Shenton, J., Dyer, T., Wiener, M., 2009. Cognitive timing: neuropsychology and anatomic basis. Brain Res. 1254, 38-48. https://doi.org/10.1016/j. brainres.2008.11.015.

Coslett, H.B., Wiener, M., Chatterjee, A., 2010. Dissociable neural systems for timing: evidence from subjects with basal ganglia lesions. PloS One 5 (4), e10324. https:// doi.org/10.1371/journal.pone.0010324.

Coull, J.T., Nobre, A.C., 2008. Dissociating explicit timing from temporal expectation with fMRI. Curr. Opin. Neurobiol. 18, 137-144. https://doi.org/10.1016/j. conb.2008.07.011.

Coull, J.T., Cheng, R.-K., Meck, W.H., 2011. Neuroanatomical and neurochemical substrates of timing. Neuropsychopharmacology 36, 3-25. https://doi.org/10.1038/ npp.2010.113.

Craig, A.D., 2002. How do you feel? Interoception: the sense of the physiological condition of the body. Curr. Opin. Neurobiol. 3, 655-666.

Crawford, J.R., Garthwaite, P.H., 2007. Comparison of a single case to a control or normative sample in neuropsychology: development of a Bayesian approach. Cogn. Neuropsychol. 24, 343-372. https://doi.org/10.1016/10.1080/ 02643290701290146.

Crawford, John R., Howell, David, 1998. Comparing an individual's test score against norms derived from small samples. Clin. Neuropsychol. 12 (4), 482-486. https://doi. org/10.1076/clin.12.4.482.7241.

Critchley, H.D., Wiens, S., Rotshtein, P., Ohman, A., Dolan, R.J., 2004. Neural systems supporting interoceptive awareness. Nat. Neurosci. 7, 189-195. https://doi.org/ 10.1038/nn1176.

Danckert, J., Ferber, S., Pun, C., Broderick, C., Striemer, C., Rock, S., Stewart, D., 2007. Neglected time: impaired temporal perception of multisecond intervals in unilateral neglect. J. Cognit. Neurosci. 19, 1706-1720.

Dalla Barba, G., Brazzarola, M., Barbera, C., Marangoni, S., Causin, F., Bartolomeo, P., Thiebaut de Schotten, M., 2018. Different patterns of confabulation in left visuospatial neglect. Exp. Brain Res. 236, 2037-2046. https://doi.org/10.1007/s00221018-5281-8.

Doricchi, F., 2003. The anatomy of neglect without hemianopia: a key role for parietalfrontal disconnection. Neuroreport 14, 2239-2243.

Dormal, V., Dormal, G., Joassin, F., Pesenti, M., 2012. A common right fronto- parietal network for numerosity and duration processing: an fMRI study. Hum. Brain Mapp. 33, 1490-1501.

Droit-Volet, S., Wearden, J.H., Zelanti, P.S., 2015. Cognitive abilities required in time judgment depending on the temporal tasks used: a comparison of children and adults. Q. J. Exp. Psychol. 68, 2216-2242. https://doi.org/10.1080/ 17470218.2015.1012087.

Ehrlé, N., Samson, S., Baulac, M., 2001. Processing of rapid auditory information in epileptic patients with left temporal lobe damage. Neuropsychologia 39, 525-531.

Foulon, C., Cerliani, L., Kinkingnehun, S., Levy, R., Rosso, C., Urbanski, M., Volle, E., Thiebaut de Schotten, M., 2018. Advanced lesion symptom mapping analyses and implementation as BCBtoolkit. GigaScience 7 (3), 1-17. https://doi.org/10.1093/ gigascience/giy004.

Freeman, J.S., Cody, F.W.J., Schady, W., 1993. The influence of external timing cues upon the rhythm of voluntary movements in Parkinson's disease. J. Neurol. Neurosurg. Psychiatry 56, 1078-1084.

Gooch, C.M., Wiener, M., Hamilton, A.C., Coslett, H.B., 2011. Temporal discrimination of sub- and suprasecond time intervals: a voxel-based lesion mapping analysis. Front. Integr. Neurosci. 5, 1-10. https://doi.org/10.3389/fnint.2011.00059.

Grahn, J.A., Brett, M., 2009. Impairment of beat-based rhythm discrimination in Parkinson's disease. Cortex 45, 54-61. https://doi.org/10.1016/j. cortex.2008.01.005.

Grefkes, C., Fink, G.R., 2014. Connectivity-based approaches in stroke and recovery of function. Lancet Neurol. 13, 206-216. https://doi.org/10.1016/S1474-4422(13) 70264-3.

Grossi, D., Di Vita, A., Palermo, L., Sabatini, U., Trojano, L., Guariglia, C., 2014. The brain network for self-feeling: a symptom-lesion mapping study. Neuropsychologia 63, 92-98. https://doi.org/10.1016/j.neuropsychologia.2014.08.004.

Grube, M., Cooper, F.E., Chinnery, P.F., Griffiths, T.D., 2010. Dissociation of durationbased and beat-based auditory timing in cerebellar degeneration. Proc. Natl. Acad. Sci. U. S. A 107, 11597-11601. 
Harrington, D.L., Haaland, K.Y., Hermanowicz, N., 1998a. Temporal processing in the basal ganglia. Neuropsychology 12, 3-12.

Harrington, D.L., Haaland, K.Y., Knight, R.T., 1998b. Cortical networks underlying mechanisms of time perception. J. Neurosci. 18, 1085-1095.

Hinton, S.C., Harrington, D.L., Binder, J.R., Durgerian, S., Rao, S.M., 2004. Neural systems supporting timing and chronometric counting: an fMRI study. Cognit. Brain Res. 21, 183-192. https://doi.org/10.1016/j.cogbrainres.2004.04.009.

Jahanshahi, M., Jones, C.R., Dirnberger, G., Frith, C.D., 2006. The substantia nigra pars compacta and temporal processing. J. Neurosci. 26, 12266-12273.

Janssen, P., Shadlen, M.N., 2005. A representation of the hazard rate of elapsed time in macaque area LIP. Nat. Neurosci. 8, 234-241. https://doi.org/10.1038/nn1386.

Jazayeri, M., Shadlen, M.N., 2015. A neural mechanism for sensing and reproducing a time interval. Curr. Biol. 25, 2599-2609. https://doi.org/10.1016/j cub.2015.08.038.

Jones, C.R., Rosenkranz, K., Rothwell, J.C., Jahanshahi, M., 2004. The right dorsolateral prefrontal cortex is essential in time reproduction: an investigation with repetitive transcranial magnetic stimulation. Exp. Brain Res. 158, 366-372. https://doi.org/ 10.1007/s00221-004-1912-3.

Kale, E.H., Ustun, S., Cicek, M., 2019. Amygdala-prefrontal cortex connectivity increased during face discrimination but not time perception. Eur. J. Neurosci. https://doi. org/10.1111/ejn.14537.

Kamali, A., Flanders, A.E., Brody, J., Hunter, J.V., Hasan, K.M., 2014. Tracing superior longitudinal fasciculus connectivity in the human brain using high resolution diffusion tensor tractography. Brain Struct. Funct. 219, 269-281. https://doi.org/ 10.1007/s00429-012-0498-.

Koch, G., Oliveri, M., Carlesimo, G.A., Caltagirone, C., 2002. Selective deficit of time perception in a patient with right prefrontal cortex lesion. Neurology 59, 1658-1659.

Koch, G., Oliveri, M., Caltagirone, C., 2009. Neural networks engaged in milliseconds and seconds time processing: evidence from transcranial magnetic stimulation and patients with cortical or subcortical dysfunction. Phil. Trans. Biol. Sci. 364, 1907-1918. https://doi.org/10.1098/rstb.2009.0018.

Koch, G., Oliveri, M., Torriero, S., Caltagirone, C., 2003. Underestimation of time perception after repetitive transcranial magnetic stimulation. Neurology 60, 1844-1846. https://doi.org/10.1212/WNL.60.11.1844.

Lee, A.C.H., Thavabalasingam, S., Alushaj, D., Çavdaroğlu, B., Ito, R., 2020. The hippocampus contributes to temporal duration memory in the context of event sequences: a cross-species perspective. Neuropsychologia 137, 107300. https://doi. org/10.1016/j.neuropsychologia.2019.107300.

Lewis, P.A., Miall, R.C., 2002. Brain activity during non-automatic motor production of discrete multi-second intervals. Neuroreport 13, 1731-1735.

Lewis, P.A., Miall, R.C., 2003a. Distinct systems for automatic and cognitively controlled time measurement: evidence from neuroimaging. Curr. Opin. Neurobiol. 13, 250-255. https://doi.org/10.1016/s0959-4388(03)00036-9.

Lewis, P.A., Miall, R.C., 2003b. Brain activation patterns during measurement of sub- and supra-second intervals. Neuropsychologia 41, 1583-1592. https://doi.org/10.1016/ S0028-3932(03)00118-0.

Lewis, P.A., Miall, R.C., 2006. A right hemispheric prefrontal system for cognitive time measurement. Behav. Process. 71, 226-324.

Li, Y., Mo, L., Chen, Q., 2015. Differential contribution of velocity and distance to time estimation during self-initiated time-to-collision judgment. Neuropsychologia 73, 35-47. https://doi.org/10.1016/j.neuropsychologia.2015.04.017.

Macar, F., Lejeune, H., Bonnet, M., Ferrara, A., Pouthas, V., Vidal, F., Maquet, P., 2002 Activation of the supplementary motor area and of attentional networks during temporal processing. Exp. Brain Res. 142, 475-485.

Mangels, J.A., Ivry, R.B., Shimizu, N., 1998. Dissociable contributions of the prefrontal and neocerebellar cortex to time perception. Cognit. Brain Res. 7, 15-39.

Mioni, G., Mattalia, G., Stablum, F., 2013. Time perception in severe traumatic brain injury patients: a study comparing different methodologies. Brain Cognit. 81, 305-312.

Meissner, K., Wittmann, M., 2011. Body signals, cardiac awareness, and the perception of time. Biol. Psychol. 86, 289-297. https://doi.org/10.1016/j.biopsycho.2011.01.001.

Melgire, M., Ragot, R., Samson, S., Penney, T.B., Meck, W.H., Pouthas, V., 2005. Auditory/visual duration bisection in patients with left or right medial-temporal lobe resection. Brain Cognit. 58, 119-124.

Merchant, H., Harrington, D.L., Meck, W.H., 2013. Neural basis of the perception and estimation of time. Annu. Rev. Neurosci. 36, 313-336. https://doi.org/10.1146/ annurev-neuro-062012-170349.

Molinari, M., Leggio, M.G., De Martin, M., Cerasa, A., Thaut, M., 2003. Neurobiology of rhythmic motor entrainment. Ann. N. Y. Acad. Sci. 999, 313-321.

Monfort, V., Pfeuty, M., Klein, M., Brissart, H., Jonas, J., Maillard, L., 2014. Distortion of time interval reproduction in an epileptic patient with a focal lesion in the right anterior insular/inferior frontal cortices. Neuropsychologia 64, 184-194. https:// doi.org/10.1016/j.neuropsychologia.2014.09.004.

Nani, A., Manuello, J., Liloia, D., Duca, S., Costa, T., Cauda, F., 2019. The neural correlates of time: a meta-analysis of neuroimaging studies. J. Cognit. Neurosci. 31 , 1796-1826. https://doi.org/10.1162/jocn_a_01459.

Nichelli, P., Alway, D., Grafman, J., 1996. Perceptual timing in cerebellar degeneration. Neuropsychologia 34, 863-871.

Nichelli, P., Clark, K., Hollnagel, C., Grafman, J., 1995. Duration processing after frontal lobe lesions. Ann. N. Y. Acad. Sci. 769, 183-190.
Noulhiane, M., Pouthas, V., Hasboun, D., Baulac, M., Samson, S., 2007. Role of the medial temporal lobe in time estimation in the range of minutes. Neuroreport 18, 1035-1038.

Ogden, R.S., Wearden, J.H., Montgomery, C., 2014. The differential contribution of executive functions to temporal generalisation, reproduction and verbal estimation. Acta Psychol. 152, 84-94. https://doi.org/10.1016/j.actpsy.2014.07.014.

Oliveri, M., Koch, G., Salerno, S., Torriero, S., Lo Gerfo, E., Caltagirone, C., 2009. Representation of time intervals in the right posterior parietal cortex: implications for a mental time line. Neuroimage 46, 1173-1179. https://doi.org/10.1016/j. neuroimage.2009.03.042.

Pacella, V., Foulon, C., Jenkinson, P.M., Scandola, M., Bertagnoli, S., Avesani, R., Fotopoulou, A., Moro, V., Thiebaut de Schotten, M., 2019. Anosognosia for hemiplegia as a tripartite disconnection syndrome. Elife 8, e46075. https://doi.org/ 10.7554/eLife.46075.

Palombo, D.J., Keane, M.M., Verfaellie, M., 2016. Does the hippocampus keep track of time? Hippocampus 26, 372-379. https://doi.org/10.1002/hipo.22528.

Palombo, D.J., Reid, A.G., Thavabalasingam, S., Hunsberger, R., Lee, A.C.H., Verfaellie, M., 2020. The human medial temporal lobe is necessary for remembering durations within a sequence of events but not durations of individual events. J. Cognit. Neurosci. 32, 497-507. https://doi.org/10.1162/jocn_a_01489.

Palombo, D.J., Verfaellie, M., 2017. Hippocampal contributions to memory for time: evidence from neuropsychological studies. Curr. Opin. Behav. Sci. 17, 107-113.

Paton, J.J., Buonomano, D.V., 2018. The neural basis of timing: distributed mechanisms for diverse functions. Neuron 98, 687-705. https://doi.org/10.1016/j. neuron.2018.03.045.

Perbal, S., Droit-Volet, S., Isingrini, M., Pouthas, V., 2002. Relationships between agerelated changes in time estimation and age-related changes in processing speed, attention, and memory. Aging Neuropsychol. Cognit. 9, 201-216.

Perbal, S., Ehrlé, N., Samson, S., Baulac, M., Pouthas, V., 2001. Time estimation in patients with right or left medial-temporal lobe resection. Neuroreport 12, 939-942.

Picton, T.W., Stuss, D.T., Shallice, T., Alexander, M.P., Gillingham, S., 2006. Keeping time: effects of focal frontal lesions. Neuropsychologia 44, 1195-1209.

Pizzamiglio, L., Judica, A., Razzano, C., Zoccolotti, P., 1989. Toward a comprehensive diagnosis of visual-spatial disorders in unilateral brain damaged patients. Psychol. Assess. 5, 199-218.

Pouthas, V., George, N., Poline, J.-B., Pfeuty, M., VandeMoorteele, P.-F., Hugueville, L., Ferrandez, A.-M., Lehericy, S., LeBihan, D., Renault, B., 2005. Neural network involved in time perception: an fMRI study comparing long and short interval estimation. Hum. Brain Mapp. 25, 433-441. https://doi.org/10.1002/hbm.20126.

Richards, W., 1973. Time reproductions by H.M. Acta Psychol. 37, 279-282.

Robertson, L.C., Knight, R.T., Rafal, R., Shimamura, A.P., 1993. Cognitive neuropsychology is more than single-case studies. J. Exp. Psychol. Learn. Mem. Cogn. 19, 710-717. https://doi.org/10.1037/0278-7393.19.3.710.

Rojkova, K., Volle, E., Urbanski, M., Humbert, F., Dell'Acqua, F., Thiebaut de Schotten, M., 2016. Atlasing the frontal lobe connections and their variability due to age and education: a spherical deconvolution tractography study. Brain Struct. Funct. 221, 1751-1766. https://doi.org/10.1007/s00429-015-1001-3.

Rorden, C., Brett, M., 2000. Stereotaxic display of brain lesions. Behav. Neurol. 12, 191-200.

Rorden, C., Karnath, H.O., Bonilha, L., 2007. Improving lesion-symptom mapping. J. Cognit. Neurosci. 19, 1081-1088.

Schlerf, J.E., Spencer, R.M., Zelaznik, H.N., Ivry, R.B., 2007. Timing of rhythmic movements in patients with cerebellar degeneration. Cerebellum 6, 221-231.

Schwartze, M., Keller, P.E., Kotz, S.A., 2016. Spontaneous, synchronized, and corrective timing behavior in cerebellar lesion patients. Behav. Brain Res. 312, 285-293. https://doi.org/10.1016/j.bbr.2016.06.040.

Schwartze, M., Rothermich, K., Kotz, S.A., 2012. Functional dissociation of pre-SMA and SMA-proper in temporal processing. Neuroimage 60, 290-298. https://doi.org/ 10.1016/j.neuroimage.2011.11.089.

Schwartze, M., Stockert, A., Kotz, S.A., 2015. Striatal contributions to sensory timing: voxel-based lesion mapping of electrophysiological markers. Cortex 71, 332-340. https://doi.org/10.1016/j.cortex.2015.07.016.

Spinnler, H., Tognoni, G., 1987. Standardizzazione e taratura italiano di test psicologici. Ital. J. Neurol. Sci. 6 (Suppl. 8), 6-120.

Teghil, A., Boccia, M., D’Antonio, F., Di Vita, A., de Lena, C., Guariglia, C., 2019. Neural substrates of internally-based and externally-cued timing: an activation likelihood estimation (ALE) meta-analysis of fMRI studies. Neurosci. Biobehav. Rev. 96, 197-209. https://doi.org/10.1016/j.neubiorev.2018.10.003.

Teghil, A., Boccia, M., Nocera, L., Pietranelli, V., Guariglia, C., 2020a. Interoceptive awareness selectively predicts timing accuracy in irregular contexts. Behav. Brain Res. 377, 112242. https://doi.org/10.1016/j.bbr.2019.112242.

Teghil, A., Di Vita, A., D'Antonio, F., Boccia, M., 2020b. Inter-individual differences in resting-state functional connectivity are linked to interval timing in irregular contexts. Cortex 128, 254-269. https://doi.org/10.1016/j.cortex.2020.03.021.

Teki, S., Grube, M., Kumar, S., Griffiths, T.D., 2011. Distinct neural substrates of duration- based and beat-based auditory timing. J. Neurosci. 31, 3805-3812. https://doi.org/10.1523/JNEUROSCI.5561-10.2011.

Thiebaut de Schotten, M., Dell'Acqua, F., Ratiu, P., Leslie, A., Howells, H., Cabanis, E., Iba-Zizen, M.T., Plaisant, O., Simmons, A., Dronkers, N.F., Corkin, S., Catani, M., 2015. From Phineas Gage and Monsieur Leborgne to H.M.: revisiting disconnection syndromes. Cereb. Cortex 25, 4812-4827. https://doi.org/10.1093/cercor/bhv173. 
Thiebaut de Schotten, M., Tomaiuolo, F., Aiello, M., Merola, S., Silvetti, M., Lecce, F., Bartolomeo, P., Doricchi, F., 2014. Damage to white matter pathways in subacute and chronic spatial neglect: a group study and 2 single-case studies with complete virtual "in vivo" tractography dissection. Cerebr. Cortex 24, 691-706. https://doi. org/10.1093/cercor/bhs351.

Tzourio-Mazoyer, N., Landeau, B., Papathanassiou, D., Crivello, F., Etard, O., Delcroix, N., Mazoyer, B., Joliot, M., 2002. Automated anatomical labeling of activations in SPM using a macroscopic anatomical parcellation of the MNI MRI single-subject brain. Neuroimage 15, 273-289. https://doi.org/10.1006/ nimg.2001.0978.

Ustun, S., Kale, E.H., Cicek, M., 2017. Neural networks for time perception and working memory. Front. Hum. Neurosci. 11, 83. https://doi.org/10.3389/ fnhum.2017.00083.

Walsh, D., 2003. A theory of magnitude: common cortical metrics of time, space and quantity. Trends Cognit. Sci. 7, 483-488. https://doi.org/10.1016/j. tics.2003.09.002.

Wiener, M., Kanai, R., 2016. Frequency tuning for temporal perception and prediction. Curr. Opin. Behav. Sci. 8, 1-6. https://doi.org/10.1016/j.cobeha.2016.01.001.

Wiener, M., Kliot, D., Turkeltaub, P.E., Hamilton, R.H., Wolk, D.A., Coslett, H.B., 2012. Parietal influence on temporal encoding indexed by simultaneous transcranial magnetic stimulation and electroencephalography. J. Neurosci. 32, 12258-12267. https://doi.org/10.1523/JNEUROSCI.2511-12.2012.

Wiener, M., Lohoff, F.W., Coslett, H.B., 2011b. Double dissociation of dopamine genes and timing in humans. J. Cognit. Neurosci. 23, 281-282.

Wiener, M., Matell, M.S., Coslett, H.B., 2011a. Multiple mechanisms for temporal processing. Front. Integr. Neurosci. 5, 1-3. https://doi.org/10.3389/ fnint.2011.00031.

Wiener, M., Turkeltaub, P., Coslett, H.B., 2010a. The image of time: a voxel-wise metaanalysis. Neuroimage 49, 1728-1740. https://doi.org/10.1016/j. neuroimage.2009.09.064.

Wiener, M., Hamilton, R., Turkeltaub, P., Matell, M.S., Coslett, H.B., 2010b. Fast forward: supramarginal gyrus stimulation alters time measurement. J. Cognit. Neurosci. 22, 23-31. https://doi.org/10.1162/jocn.2009.21191.

Wittmann, M., Simmons, A.N., Aron, J.L., Paulus, M.P., 2010. Accumulation of neural activity in the posterior insula encodes the passage of time. Neuropsychologia 48, 3110-3120. https://doi.org/10.1016/j.neuropsychologia.2010.06.023.

Wittmann, M., Simmons, A.N., Flagan, T., Lane, S.D., Wackermann, J., Paulus, M.P., 2011. Neural substrates of time perception and impulsivity. Brain Res. 1406, 43-58. https://doi.org/10.1016/j.brainres.2011.06.048. 